\title{
Concomitant loss of SMARCA2 and SMARCA4 expression in small cell carcinoma of the ovary, hypercalcemic type
}

\author{
Petar Jelinic $^{1}$, Brooke A Schlappe ${ }^{1}$, Niamh Conlon ${ }^{2}$, Jill Tseng ${ }^{1}$, Narciso Olvera ${ }^{1}$, \\ Fanny Dao ${ }^{1}$, Jennifer J Mueller ${ }^{1}$, Yaser Hussein ${ }^{2}$, Robert A Soslow ${ }^{2}$ and Douglas A Levine ${ }^{1}$ \\ ${ }^{1}$ Department of Surgery, Gynecology Research Laboratory, Gynecology Service, Memorial Sloan Kettering \\ Cancer Center, New York, NY, USA and ${ }^{2}$ Department of Pathology, Memorial Sloan Kettering Cancer Center, \\ New York, NY, USA
}

\begin{abstract}
Small cell carcinoma of the ovary, hypercalcemic type is an aggressive tumor generally affecting young women with limited treatment options. Mutations in SMARCA4, a catalytic subunit of the SWI/SNF chromatin remodeling complex, have recently been identified in nearly all small cell carcinoma of the ovary, hypercalcemic type cases and represent a signature molecular feature for this disease. Additional biological dependencies associated with small cell carcinoma of the ovary, hypercalcemic type have not been identified. SMARCA2, another catalytic subunit of the SWI/SNF complex mutually exclusive with SMARCA4, is thought to be post-translationally silenced in various cancer types. We analyzed $\mathbf{1 0}$ archival small cell carcinoma of the ovary, hypercalcemic type cases for SMARCA2 protein expression by immunohistochemistry and found that SMARCA2 expression was lost in all but one case. None of the $\mathbf{5 0}$ other tumors that primarily or secondarily involved the ovary demonstrated concomitant loss of SMARCA2 and SMARCA4. Deep sequencing revealed that this loss of SMARCA2 expression is not the result of mutational inactivation. In addition, we established a small cell carcinoma of the ovary, hypercalcemic type patient-derived xenograft and confirmed the loss of SMARCA2 in this in vitro model. This patient-derived xenograft model, established from a recurrent tumor, also had unexpected mutational features for this disease, including functional mutations in TP53 and POLE. Taken together, our data suggest that concomitant loss of SMARCA2 and SMARCA4 is another hallmark of small cell carcinoma of the ovary, hypercalcemic type-a finding that offers new opportunities for therapeutic interventions.

Modern Pathology (2016) 29, 60-66; doi:10.1038/modpathol.2015.129; published online 13 November 2015
\end{abstract}

Small cell carcinoma of the ovary, hypercalcemic type is a rare and aggressive subtype of ovarian cancer that affects mainly young women. The majority of patients die from this disease within 2 years, with long-term survival largely limited to patients with tumors confined to the ovary. ${ }^{1}$ There are currently no effective therapies to treat this disease, and histological classification has been challenging. Recent studies have uncovered inactivating SMARCA4 germline and somatic mutations in nearly all small cell carcinoma of the ovary, hypercalcemic type cases, suggesting that loss of SMARCA4 is a signature feature for this disease. ${ }^{2-4}$ Further evaluation of the molecular landscape

Correspondence: Dr DA Levine, MD, Department of Surgery, Gynecology Research Laboratory, Gynecology Service, Memorial Sloan Kettering Cancer Center, 1275 York Avenue, New York, NY 10065, USA.

E-mail: levine2@mskcc.org

Received 1 August 2015; revised 25 September 2015; accepted 26 September 2015; published online 13 November 2015 beyond mutational studies is important to gain a deeper understanding of the disease mechanisms and explore alternative therapeutic approaches for treatment.

SMARCA4, also known as BRG1, is a catalytic subunit of the SWI/SNF chromatin remodeling complex. It has a major role in rendering chromatin accessible to proteins essential for the regulation of gene expression, resulting mostly in activation of target genes. Subunits of the SWI/SNF complex are altered in $>20 \%$ of all cancers, with SMARCA4 loss being one of the most frequent alterations among the subunits. ${ }^{5}$ It is well documented that in the majority of cancer cells, the loss of SMARCA4 results in aggressive growth, suggesting it normally functions as a tumor suppressor. ${ }^{5,6}$

In non-small cell lung carcinoma cell lines with loss of SMARCA4 expression, cell viability is dependent on retained expression of SMARCA2 (also known as BRM), a mutually exclusive catalytic subunit of the SWI/SNF complex. ${ }^{7-9}$ BIN67 is a small cell carcinoma of the ovary, hypercalcemic type 
SMARCA4-mutant cell line with minimal expression levels of SMARCA2, ${ }^{3}$ suggesting that in small cell carcinoma of the ovary, hypercalcemic type cells SMARCA2 expression may not be required for cell survival. These observations suggest that SMARCA4-mutant cancers may not be universally dependent on SMARCA2 expression, implying that the interdependency between these two subunits is complex and likely contextual. ${ }^{10}$ Recent studies have suggested that SMARCA4 immunohistochemistry may be useful to distinguish small cell carcinoma of the ovary, hypercalcemic type from potential diagnostic mimics. ${ }^{11}$

To better understand the relationship between SMARCA2 and SMARCA4 in the context of small cell carcinoma of the ovary, hypercalcemic type, we evaluated SMARCA2 expression in 10 small cell carcinoma of the ovary, hypercalcemic type cases and identified concomitant loss with SMARCA4. We also confirmed SMARCA2 loss in a small cell carcinoma of the ovary, hypercalcemic type patient-derived xenograft with an unexpected molecular landscape.

\section{Materials and methods}

\section{Patients and Tumor Samples}

In this study, we included 10 archival cases, of which 9 (cases 101-103, 106, and 108-112) have been previously reviewed and analyzed for SMARCA4 mutations. ${ }^{2}$ We also included two new cases, one additional archival case (114) and a newly established case from a patient-derived xenograft model (113). Using previously described guidelines, specialty gynecologic pathologists reviewed two new cases-113 and 114-to confirm the diagnosis of small cell carcinoma of the ovary, hypercalcemic type. Clinical data collection was limited to only age and year of diagnosis owing to the Health Insurance Portability and Accountability Act regulations. The patient-derived xenograft case (113) was collected from a patient undergoing an excisional biopsy for recurrent disease. DNA was extracted from formalin-fixed paraffin-embedded tumors with at least $50 \%$ tumor cell nuclei according to standard protocols (DNeasy Blood\&Tissue kit; Qiagen \#69506).

\section{MSK-IMPACT Assay and Sequencing}

The MSK-IMPACT assay was performed as previously described, ${ }^{2}$ with the following modifications. Barcoded sequence libraries were prepared using $250 \mathrm{ng}$ of genomic DNA (Kapa Biosystems, Wilmington, MA, USA) and combined into a single equimolar pool. The captured pool was subsequently sequenced on an Illumina HiSeq 2500 sequencer (Illumina, San Diego, CA, USA) as paired-end 100base pair reads, producing at least 250 -fold coverage per tumor. A panel of 341 known oncogenes and tumor-suppressor genes that are frequently altered in cancer were sequenced (Supplementary Table S1). The SMARCA4 mutations were validated using Sanger sequencing. Primers were specifically designed to amplify the mutations of interest. Sequencing of the coding regions and splice sites for SMARCA2 was done using AmpliSeq. The mean coverage was $3730 \mathrm{X}$ for $99.6 \%$ of the coding region (primer details are available upon request).

\section{Immunoblotting}

Tumor samples from frozen tissue were available for cases 101, 102, and 113 (patient-derived xenograft tumor). Before protein extraction, hematoxylin and eosin-stained frozen section slides were prepared from this tissue and examined by a gynecologic pathologist to ensure tumor purity. The proteins were extracted using RIPA Lysis buffer supplemented with protease and phosphatase inhibitors. After 15 min of incubation on ice, the lysates were spun for $15 \mathrm{~min}$ at $20000 \mathrm{~g}$ in the cold Microfuge. Protein concentrations were measured by Bradford. Extracted proteins were resolved on SDS-PAGE, transferred onto nitrocellulose, and blotted for antibodies targeting proteins of interest: SMARCA4 (Santa Cruz Biotechnology, Dallas, TX, USA; \#sc-10768, dilution 1:1000), SMARCA2 (Cell Signaling Technology, Danvers, MA, USA; \#11966, dilution 1:1000), and $\beta$-Actin (Santa Cruz Biotechnology, sc-69879, dilution 1:10 000).

\section{Immunohistochemistry}

All assays were performed on formalin-fixed paraffin-embedded slides. Immunohistochemistry staining for SMARCA4 was performed as previously described. ${ }^{2}$ The immunohistochemistry method for SMARCA2 was optimized as follows: heat steaming with EDTA at $\mathrm{pH} 8$ for $30 \mathrm{~min}$ resulted in epitope retrieval. Antigen retrieval followed by overnight incubation at $4^{\circ} \mathrm{C}$ with primary antibody targeting SMARCA2 (Sigma-Aldrich, St. Louis, MO, USA; \#HPA029981, dilution 1:1000). Biotinylated antiRabbit IgG (Vector Laboratories, Burlingame, CA, USA; \#BA-100, dilution 1:1000) and ABC (Vector Laboratories; \#PK-6100) were used to detect the bound antibody. Diaminobenzidine was used as the chromogen. Absence of nuclear staining in tumor cells in the presence of internal positive control (blood vessels and stromal cells) was scored as 'loss of expression'.

\section{Cell Lines and Overexpression Studies}

The small cell carcinoma of the ovary, hypercalcemic type BIN67 model cell line was generously gifted by $\mathrm{Dr}$ Antony $\mathrm{N}$ Karnezis (British Columbia Cancer Agency, Vancouver, Canada). The cells were 

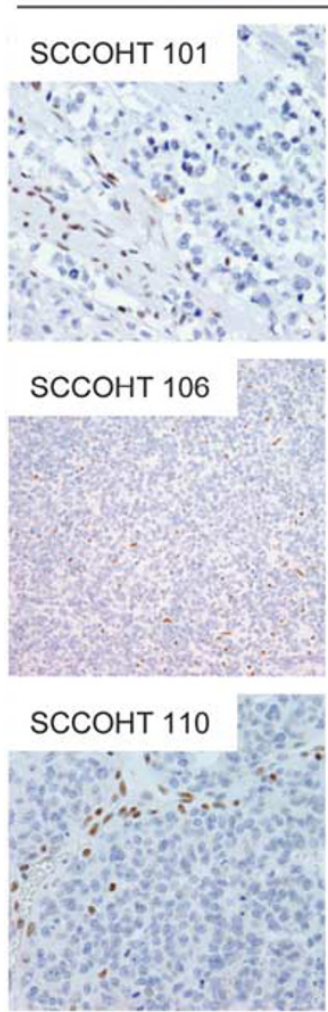

SMARCA2
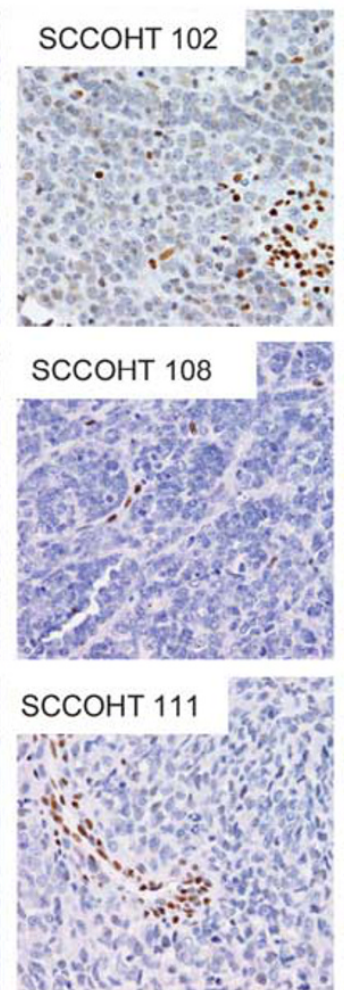

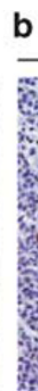

b SMARCA2
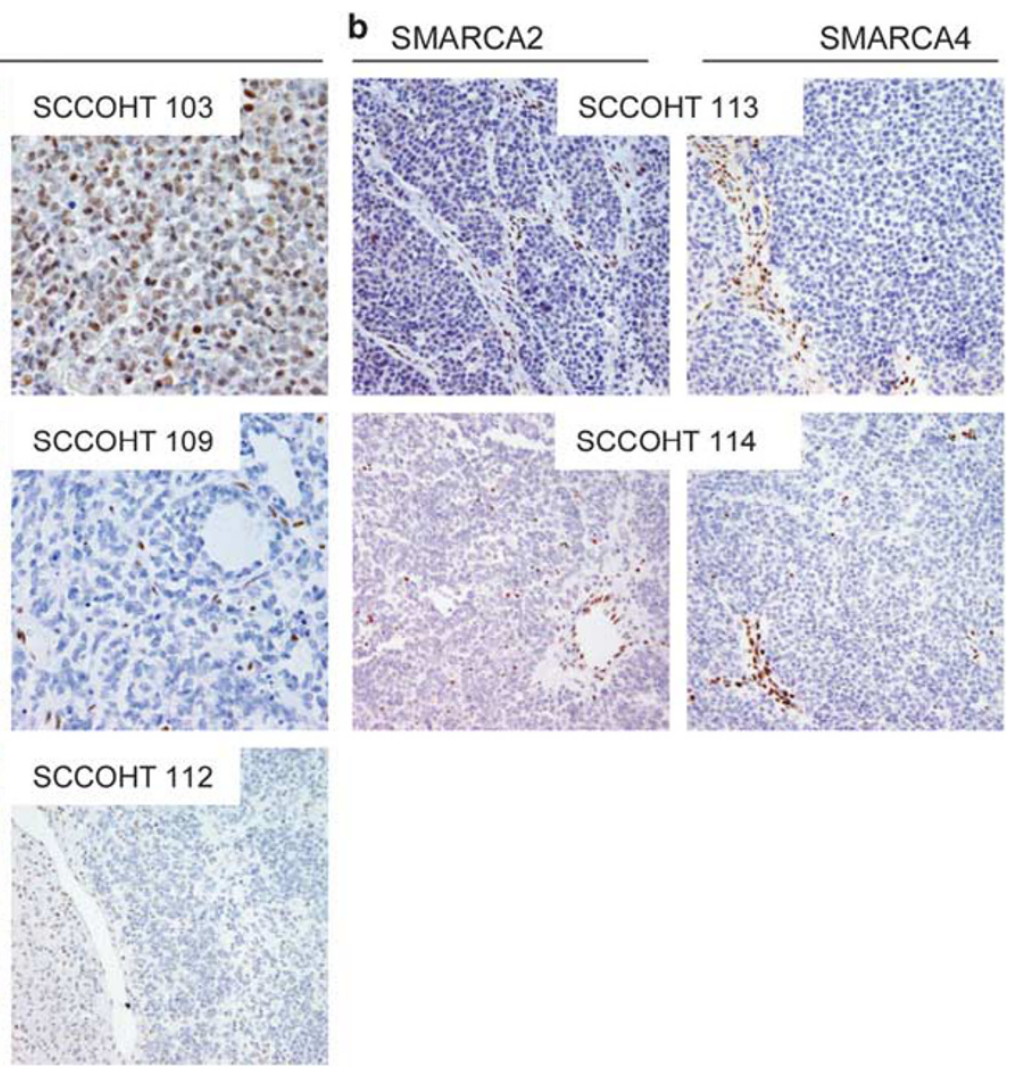

Figure 1 Immunohistochemistry for SMARCA2 and SMARCA4 in formalin-fixed paraffin-embedded small cell carcinoma of the ovary, hypercalcemic type cases. (a) SMARCA2 staining of the original small cell carcinoma of the ovary, hypercalcemic type cases previously described and stained for SMARCA4. Note the strong staining of stromal cell nuclei as internal control. (b) SMARCA2 and SMARCA4 staining for two new small cell carcinoma of the ovary, hypercalcemic type cases.

cultured in DME-HG media supplemented with 10\% FCS under the standard tissue culture conditions. The non-small cell lung carcinoma H1299 cell line was obtained from the American Type Culture Collection. The cells were cultured in RPMI-HG media supplemented with $10 \%$ FCS under the standard tissue culture conditions. The expression plasmid containing cDNA for SMARCA4 (OriGene Technologies, Rockville, MD, USA; SC323288) was transiently transfected into BIN67 and H1299 cells using FuGENE protocol according to the manufacturer's suggestions (Promega Corporation, Madison, WI, USA; E2311).

\section{Results}

Analysis of SMARCA2 expression by immunohistochemistry in small cell carcinoma of the ovary, hypercalcemic type cases revealed loss of SMARCA2 expression in $9(90 \%)$ of 10 archival cases (Figure 1, Table 1). For the three cases with available frozen tissue, we also confirmed the loss of SMARCA2 protein expression by immunoblotting (Figure 2a). To investigate whether this loss of expression was owing to mutations in the SMARCA2 gene, we performed deep sequencing of the coding region and splice sites of SMARCA2. No SMARCA2 mutations were identified, suggesting that the loss of protein expression is non-mutational.

The only case (\#103) with normal expression of SMARCA2 was also the only case that expressed SMARCA4 in our previous report. ${ }^{2}$ This suggests that SMARCA2 and SMARCA4 could be interdependently expressed in small cell carcinoma of the ovary, hypercalcemic type. To explore this relationship further, we re-introduced SMARCA4 into BIN67 small cell carcinoma of the ovary, hypercalcemic type and H1299 non-small cell lung carcinoma cell lines that lack the expression of SMARCA4 (Figure 2b). Though H1299 cells express SMARCA2 normally, BIN67 cells have very low levels of SMARCA2 expression. We did not observe an increased expression of SMARCA2 upon SMARCA4 overexpression, suggesting that the presence of SMARCA4 alone is not sufficient for SMARCA2 re-expression in small cell carcinoma of the ovary, hypercalcemic type.

We previously reported SMARCA4 mutations in 9 of the 10 archival small cell carcinoma of the ovary, hypercalcemic type cases included in this study. ${ }^{2}$ Here, we also report the loss of SMARCA4 expression in two new cases: 113 and 114 (Figure 1b). Case 113 was a patient-derived xenograft obtained in the 
Table 1 Summary of SMARCA2 and SMARCA4 mutation and immunohistochemistry status in small cell carcinoma of the ovary, hypercalcemic type cases

SMARCA4

Case number

Age at diagnosis (years)

\section{Gene mutations}

Protein change

\begin{tabular}{lc}
\hline $101^{\mathrm{a}}$ & 40 \\
$102^{\mathrm{a}}$ & 22 \\
$103^{\mathrm{a}}$ & 19 \\
$106^{\mathrm{a}}$ & 40 \\
$108^{\mathrm{a}}$ & 22 \\
$109^{\mathrm{a}}$ & 32 \\
$110^{\mathrm{a}}$ & 42 \\
$111^{\mathrm{a}}$ & 35 \\
$112^{\mathrm{a}}$ & 28 \\
113 & $\mathrm{NA}$ \\
114 & 27 \\
\hline
\end{tabular}

SMARCA2

\begin{tabular}{ll}
\hline & \\
Gene status & IHC \\
& \\
\hline Wild type & Loss \\
Wild type & Loss \\
Wild type & Retained \\
NA & Loss \\
NA & Loss \\
NA & Loss \\
NA & Loss \\
Wild type & Loss \\
Wild type & Loss \\
Wild type & Loss \\
Wild type & Loss \\
\end{tabular}

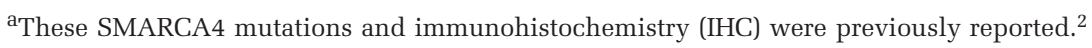

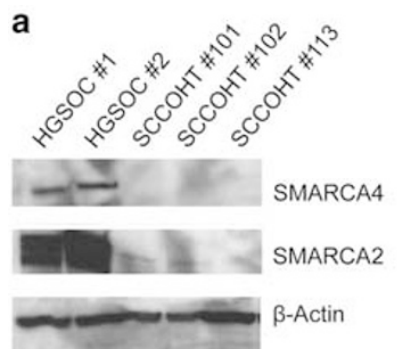

b

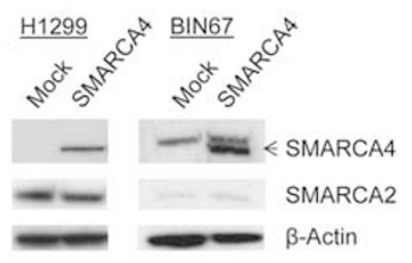

Figure 2 (a) Concomitant loss of SMARCA2 and SMARCA4 expression in small cell carcinoma of the ovary, hypercalcemic type measured by immunoblotting. Two high-grade serous ovarian carcinoma (HGSOC\#1 and \#2) cases were used as a positive control. (b) SMARCA4 overexpression in H1299 non-small cell lung carcinoma and BIN67 small cell carcinoma of the ovary, hypercalcemic type cell lines. Note the upper band in BIN67 cells blotted for SMARCA4 is non-specific. $\beta$-Actin is the loading control.

setting of recurrent small cell carcinoma of the ovary, hypercalcemic type. The xenograft tumor was grown in the peritoneum of SCID mice, as previously described, before being explanted and analyzed for mutations and protein expression. ${ }^{12}$ The tumor demonstrated characteristic features of small cell carcinoma of the ovary, hypercalcemic type, including a combination of small neoplastic and larger rhabdoid cells (Figure 3a). Case 114 was from a 27year-old woman with newly diagnosed small cell carcinoma of the ovary, hypercalcemic type. These cases were deep-sequenced using the MSK-IMPACT assay, and Sanger sequencing confirmed the results. Biallelic SMARCA4 mutations, the small cell carcinoma of the ovary, hypercalcemic type molecular signature feature, were present in both cases (Figure $3 \mathrm{~b}$ and Supplementary Figure S1). Immunohistochemistry staining confirmed the loss of SMARCA4 expression.
Surprisingly, case 113 had a number of additional mutations previously not reported in small cell carcinoma of the ovary, hypercalcemic type (Figure $3 \mathrm{~b}$ and Supplementary Table S2). As matched normal samples were unavailable for these cases, we cannot rule out the germline nature of the heterozygous missense variants. We used Mutation Assessor from the cBioPortal for Cancer Genomics (cbioportal.mskcc.org) to assess the functional impact of identified mutations. The TP53 and POLE mutations in case 113 were predicted to be functional. The functional significance of the TP53 I254N homozygous missense mutation was confirmed by immunohistochemistry, demonstrating overexpression of TP53 protein (Figure 3c). Another unexpected alteration was the heterozygous D287A mutation in the DNA polymerase POLE, a feature of hypermutated cancers. ${ }^{13}$ Given that this mutation is adjacent to the common hot spot mutation P286H/R, the most frequent of all POLE mutations, ${ }^{14}$ and it is within the POLE's essential exonuclease domain, it is likely that the D287A mutation is critical to protein function.

The coordinated expression of SMARCA2 and SMARCA4 protein (loss of both in 10 cases and retention of both in one case) is found in $100 \%$ of the small cell carcinoma of the ovary, hypercalcemic type cases tested, suggesting that this is a signature feature for this disease. To further demonstrate that this is specific to small cell carcinoma of the ovary, hypercalcemic type, we stained 50 additional cases of various tumor types that primarily or secondarily involved the ovary (10 small cell lung carcinomas, 20 ovarian clear cell carcinomas, 10 ovarian granulosa cell tumors, and 10 melanomas) with SMARCA2 and SMARCA4 antibodies (Supplementary Figure S2 and Supplementary Table S3). Forty-eight (96\%) of 50 cases showed normal expression of SMARCA4. The two cases with absence of SMARCA4 

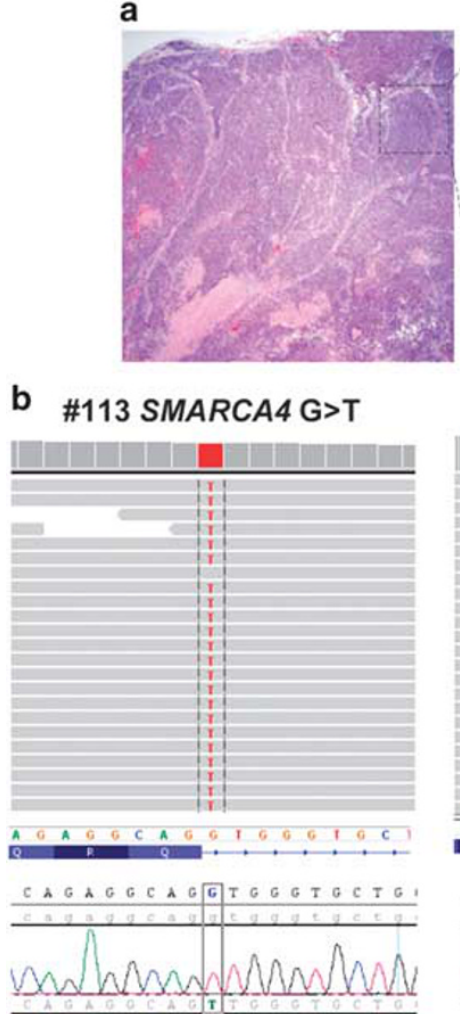

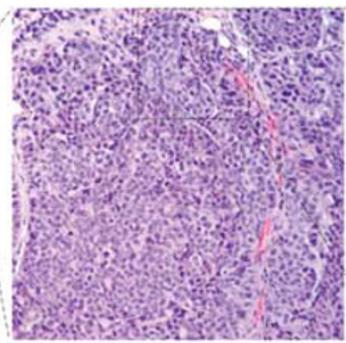

\#113 POLE A>T

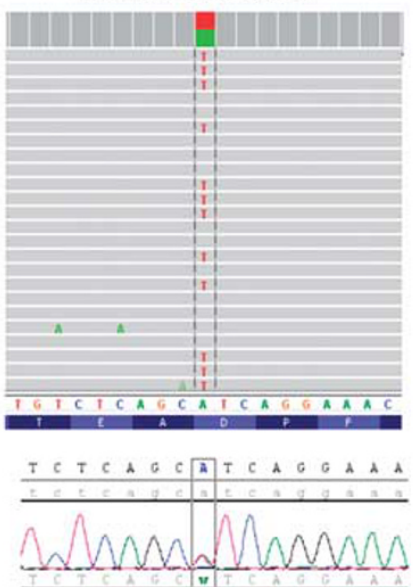

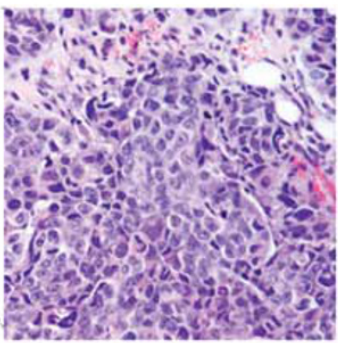

\#113 TP53 A>T
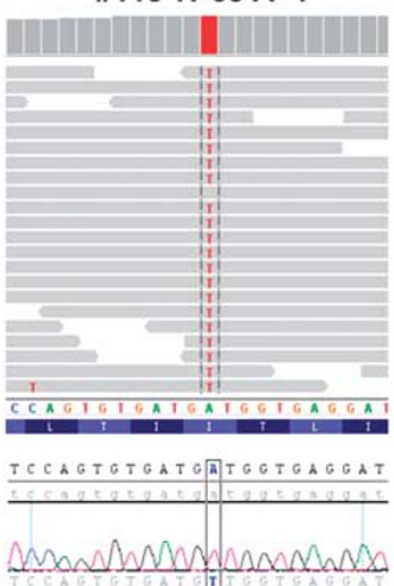

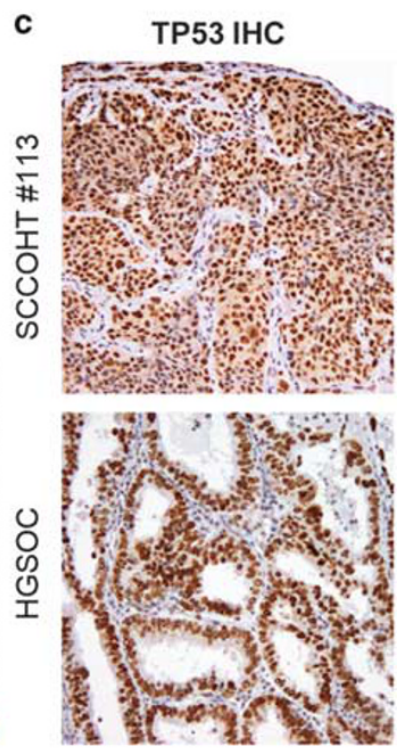

Figure 3 Assessment of the small cell carcinoma of the ovary, hypercalcemic type patient-derived xenograft case 113. (a) Hematoxylin and eosin staining shows histopathological features of the explanted small cell carcinoma of the ovary, hypercalcemic type. (b) Sequence analyses of SMARCA4, POLE and TP53. Top panels: next-generation sequence coverage demonstrating identified variants. Bottom panels: validation by Sanger sequencing. (c) TP53 immunohistochemical staining. High-grade serous ovarian carcinoma was used as a positive control.

expression, one ovarian clear cell carcinoma (OCCC\#20) and one metastatic melanoma (Mel\#32), had retained SMARCA2 expression. Only $1(2 \%)$ of 50 cases, an ovarian clear cell carcinoma, (OCCC\#18), showed absence of SMARCA2 expression; yet, this case retained SMARCA4 expression. These data further confirm the unique findings of concomitant loss of SMARCA2 and SMARCA4 in small cell carcinoma of the ovary, hypercalcemic type.

\section{Discussion}

In this report, we identified concomitant loss of SMARCA2 and SMARCA4 expression in nine small cell carcinoma of the ovary, hypercalcemic type formalin-fixed paraffin-embedded cases and one patient-derived xenograft model. We confirm that the absence of SMARCA2 expression is not a result of germline or somatic mutations. This is in agreement with previously reported studies demonstrating the lack of SMARCA2 mutations in SMARCA2-deficient pancreatic and lung cancer cell lines, suggesting that loss of SMARCA2 may be a result of epigenetic or post-translational modifications. ${ }^{15-17}$ Recently, it has been reported that SMARCA2 expression is regulated by histone deacetylases HDAC3 and HDAC9, in addition to transcription factors MEF2D and GATA $3 .^{18}$ Also, overexpression of HDAC9 has been shown to correlate with decreased SMARCA2 expression, whereas HDAC9 depletion causes elevated SMARCA2 expression. Therefore, it is plausible that small cell carcinoma of the ovary, hypercalcemic type has increased expression of HDAC9, resulting in low SMARCA2 expression. In addition, it has been reported that SMARCA2 promoter polymorphism contributes to differential expression in lung cancer cell lines and primary lung cancers, where the presence of two insertions within the promoter correlates with SMARCA2 loss of expression. ${ }^{19}$ The presence of these insertions within the SMARCA2 promoter in small cell carcinoma of the ovary, hypercalcemic type could explain loss of SMARCA2 expression in this disease. Though SMARCA2 promoter methylation has not been described as a major epigenetic alteration in cancers, further study will be required to exclude this potential mechanism.

The only small cell carcinoma of the ovary, hypercalcemic type case (\#103) in this series that showed normal SMARCA2 expression was also the only one that had strong SMARCA4 expression. We 
confirmed that the presence of SMARCA4 alone is not sufficient for SMARCA2 re-expression in small cell carcinoma of the ovary, hypercalcemic type. Case 103 has a non-functional helicase domain as a result of a 2 exon in-frame deletion despite retaining SMARCA4 expression; ${ }^{2}$ however, the protein is still expressed. Even though unlikely, it is possible that this truncated helicase-dead SMARCA4 may contribute to re-expression of SMARCA2.

Unique from our other cases, case 113 was not only a patient-derived xenograft, but it also originated from recurrent small cell carcinoma of the ovary, hypercalcemic type disease. In addition to the expected SMARCA4 mutation, we identified high confidence mutations in POLE and TP53. The POLE mutation may give rise to other mutations owing to the mutator phenotype seen with POLE exonuclease domain mutations; however, owing to the lack of a matched normal DNA sample, germline variants cannot be excluded. This case is also the only recurrent case in our cohort, raising the possibility that other mutations may have occurred as a result of tumor progression or drug treatment. Examination of the original tumor from the time of diagnosis, which is not available at present, could help determine the sequence of mutation evolution.

Concomitant absence of SMARCA2 and SMARCA4 has been reported in a subset $(10 \%)$ of non-small cell lung carcinoma cases. ${ }^{20}$ These nonsmall cell lung carcinoma cases had a worse overall survival compared with cases that did not have concomitant absence of SMARCA2 and SMARCA4. This suggests that the loss of both SMARCA2 and SMARCA4 may contribute to the aggressiveness of small cell carcinoma of the ovary, hypercalcemic type. Our data further suggest that the absence of SMARCA2 expression in small cell carcinoma of the ovary, hypercalcemic type is non-mutational and potentially the result of epigenetic or post-translational silencing. The reversal of epigenetic silencing of SMARCA2 and its resultant re-expression is a potential therapeutic approach for cancers with a SMARCA2-depleted background. ${ }^{17,21,22}$ SMARCA2 expression may be restored with HDAC inhibitors and result in reduced cell growth. ${ }^{15,17,18}$ Finally, a recent report suggests that flavonoids are able to restore SMARCA2 expression, ${ }^{23}$ proposing that this group of drugs could be effective in cancers in which re-expression of SMARCA2 results in growth retardation. Reversal of epigenetic silencing of SMARCA2 could be further explored as a treatment option for patients with small cell carcinoma of the ovary, hypercalcemic type. The near universal mutations in SMARCA4 coupled with newly discovered concomitant loss of SMARCA2 expression substantiates the designation of small cell carcinoma of the ovary, hypercalcemic type as a fascinating rare tumor.

\section{Acknowledgments}

We thank Paul Haluska and Marc A Becker from Mayo Clinic for engrafting the patient-derived xenograft tumor. We are grateful to all the small cell carcinoma of the ovary, hypercalcemic type patients who have shared their biospecimens with us. Cycle for Survival of Memorial Sloan Kettering Cancer Center, Katie Oppo Research Fund, Arnold Chavkin and Laura Chang, Laura Mercier Foundation, Department of Defense CDMRP Grant W81XWH-11-2-0230, NIH/NCI Grant P30CA008748.

\section{Disclosure/conflict of interest}

The authors declare no conflict of interest.

\section{References}

1 Young RH, Oliva E, Scully RE. Small cell carcinoma of the ovary, hypercalcemic type. A clinicopathological analysis of 150 cases. Am J Surg Pathol 1994;18: 1102-1116.

2 Jelinic $\mathrm{P}$, Mueller JJ, Olvera $\mathrm{N}$ et al. Recurrent SMARCA4 mutations in small cell carcinoma of the ovary. Nat Genet 2014;46:424-426.

3 Witkowski L, Carrot-Zhang J, Albrecht S et al. Germline and somatic SMARCA4 mutations characterize small cell carcinoma of the ovary, hypercalcemic type. Nat Genet 2014;46:438-443.

4 Ramos P, Karnezis AN, Craig DW et al. Small cell carcinoma of the ovary, hypercalcemic type, displays frequent inactivating germline and somatic mutations in SMARCA4. Nat Genet 2014;46:427-429.

5 Kadoch C, Hargreaves DC, Hodges C et al. Proteomic and bioinformatic analysis of mammalian SWI/SNF complexes identifies extensive roles in human malignancy. Nat Genet 2013;45:592-601.

6 Wilson BG, Roberts CW. SWI/SNF nucleosome remodellers and cancer. Nat Rev Cancer 2011;11:481-492.

7 Oike T, Ogiwara H, Tominaga Y et al. A synthetic lethality-based strategy to treat cancers harboring a genetic deficiency in the chromatin remodeling factor BRG1. Cancer Res 2013;73:5508-5518.

8 Hoffman GR, Rahal R, Buxton F et al. Functional epigenetics approach identifies BRM/SMARCA2 as a critical synthetic lethal target in BRG1-deficient cancers. Proc Natl Acad Sci USA 2014;111:3128-3133.

9 Wilson BG, Helming KC, Wang X et al. Residual complexes containing SMARCA2 (BRM) underlie the oncogenic drive of SMARCA4 (BRG1) mutation. Mol Cell Biol 2014;34:1136-1144.

10 Marquez SB, Thompson KW, Lu L et al. Beyond mutations: additional mechanisms and implications of SWI/SNF complex inactivation. Front Oncol 2014;4:372.

11 Karanian-Philippe M, Velasco V, Longy $\mathrm{M}$ et al. SMARCA4 (BRG1) loss of expression is a useful marker for the diagnosis of ovarian small cell carcinoma of the hypercalcemic type (ovarian rhabdoid tumor): a comprehensive analysis of 116 rare gynecologic tumors, 9 soft tissue tumors, and 9 melanomas. Am J Surg Pathol 2015;39:1197-1205. 
12 Weroha SJ, Becker MA, Enderica-Gonzalez S et al. Tumorgrafts as in vivo surrogates for women with ovarian cancer. Clin Cancer Res 2014;20:1288-1297.

13 Cancer Genome Atlas Research N, Kandoth C, Schultz $\mathrm{N}$ et al. Integrated genomic characterization of endometrial carcinoma. Nature 2013;497:67-73.

14 Kane DP, Shcherbakova PV. A common cancerassociated DNA polymerase epsilon mutation causes an exceptionally strong mutator phenotype, indicating fidelity defects distinct from loss of proofreading. Cancer Res 2014;74:1895-1901.

15 Yamamichi N, Yamamichi-Nishina M, Mizutani T et al. The Brm gene suppressed at the post-transcriptional level in various human cell lines is inducible by transient HDAC inhibitor treatment, which exhibits antioncogenic potential. Oncogene 2005;24:5471-5481.

16 Reisman D, Glaros S, Thompson EA. The SWI/SNF complex and cancer. Oncogene 2009;28:1653-1668.

17 Glaros S, Cirrincione GM, Muchardt C et al. The reversible epigenetic silencing of BRM: implications for clinical targeted therapy. Oncogene 2007;26: 7058-7066.

18 Kahali B, Gramling SJ, Marquez SB et al. Identifying targets for the restoration and reactivation of BRM. Oncogene 2014;33:653-664.

19 Liu G, Gramling S, Munoz D et al. Two novel BRM insertion promoter sequence variants are associated with loss of BRM expression and lung cancer risk. Oncogene 2011;30:3295-3304.
20 Reisman DN, Sciarrotta J, Wang W et al. Loss of BRG1/ BRM in human lung cancer cell lines and primary lung cancers: correlation with poor prognosis. Cancer Res 2003;63:560-566

21 Gramling S, Rogers C, Liu G et al. Pharmacologic reversal of epigenetic silencing of the anticancer protein BRM: a novel targeted treatment strategy. Oncogene 2011;30:3289-3294.

22 Gramling S, Reisman D.. Discovery of BRM targeted therapies: novel reactivation of an anti-cancer gene. Lett Drug Des Discov 2011;8:93-99.

23 Kahali B, Marquez SB, Thompson KW et al. Flavonoids from each of the six structural groups reactivate BRM, a possible cofactor for the anticancer effects of flavonoids. Carcinogenesis 2014;35: 2183-2193.

(c) (i) $(\Theta$ This work is licensed under a Creative Commons Attribution-NonCommercialNoDerivs 4.0 International License. The images or other third party material in this article are included in the article's Creative Commons license, unless indicated otherwise in the credit line; if the material is not included under the Creative Commons license, users will need to obtain permission from the license holder to reproduce the material. To view a copy of this license, visit http://creativecommons.org/licenses/ by-nc-nd/4.0/

Supplementary Information accompanies the paper on Modern Pathology website (http://www.nature.com/ modpathol) 